Eur Neurol 2007;57:127-128

DOI: $10.1159 / 000098104$

\section{Prognostic Relevance of Early MRI Vessel Signs Is Highly Overrated - Part 2}

Peter D. Schellinger Martin Köhrmann

Department of Neurology, University of Erlangen, Erlangen, Germany

In this issue, Girot et al. [1] present a series of 30 consecutive stroke patients imaged with MRI, including FLAIR sequences, within $12 \mathrm{~h}$ after onset of symptoms. Their aim was to test for a possible association of FLAIR hyperintense vessel signs (FLAIR HVS) with a worse outcome as measured by a dependent or dead outcome according to the modified Rankin Scale (mRS 3-6) at 1 month. They found that $12 / 13$ patients with as opposed to $4 / 17$ patients without distal FLAIR HVS had a poor outcome. No patient was treated with rt-PA. The authors conclude that distal HVS predicts worse outcome in ischemic stroke.

Two major points come to mind when analyzing the authors' findings. First, the high sensitivity of FLAIR HVS in distal and proximal occlusions according to TOF MRA in this patient group when compared with other analyses. This may be explained by the open, non-blinded assessment by 2 examiners in a consensus session as opposed to a fully blinded rating process [2], a mistake also made by others [3], which has been discussed before [4]. Differences in sensitivity are not explained by the fact whether or not patients receive rt-PA: TOF and FLAIR HVS should always show the same images regardless of therapy as both image the same phenomenon, i.e. slow flow and clot/occlusion. A study twice the size could not find any prognostic value of FLAIR HVS with regard to recanalization, outcome, hemorrhage and response pattern to intravenous rt-PA [2]. Again, with regard to the authors' objective these populations are not different because of rt-PA treatment. Liebeskind [5] also commented that HVS represents areas with slow blood flow and therefore may indicate the location and extent of compensatory distal collateral flow, which is a good thing.

Second, there are so many major imbalances between groups that the statistical analysis with a bivariate test is not suitable, in fact plainly wrong, at least if valid conclusions are to be drawn. The authors compare patients with severe strokes (old, high NIHSS, DWI lesion, vessel occlusion) to patients who mostly have no or only a minimal stroke. They prove the obvious, comparable to stating that it is better to be rich and healthy than poor and sick.
Outcome in stroke (usually assessed after 3 months and not 1 month after symptom onset) is driven by three clear predictors and maybe a few secondary ones, depending on which analyses in the literature one looks at. Age and baseline severity as measured by the NIHSS are the strongest predictors of outcome. NIHSS correlates with the presence of a proximal vessel occlusion. Occurrence of symptomatic ICH (with or without thrombolysis) is a third strong predictor for stroke outcome. Weaker but possibly associated variables are fever and hyperglycemia. In theory, if HVS were an indicator of vessel occlusion with high diagnostic accuracy (which it is not and neither did the authors appropriately assess this), HVS could be a predictor for a worse outcome. However, in this study patients with a poor outcome are 10 years older (68 vs. 58 years, apparent but not significant difference due to low numbers), have a significantly higher NIHSS (16 vs. 5 points, $\mathrm{p}=0.001$ ) and have significantly more often a proximal vessel occlusion ( 9 vs. 1 patients), which most likely is the reason for the difference in NIHSS. They are also more likely to have a DWI lesion $(13 / 13$ vs. $12 / 17, \mathrm{p}=0.052)$. The authors did not correct for these imbalances because they believe that the number of patients is somewhat small for multivariate analysis, which is partly true. However, depending on the question they had set out to answer, for 1 independent variable to be tested 5-10 observations (patients) are sufficient. You could enter all your variables with trend $(\mathrm{p}<0.15)$ and significance $(\mathrm{p}<0.05)$ into a logistic regression: NIHSS, TOF occlusion (distal and proximal), DWI lesion present, age. Even if FLAIR HVS turned out to be an independent predictor of outcome (which I doubt), this finding would be weakened by a cocorrelation with TOF findings and the fact that we are dealing with non-blinded data.

The presence of FLAIR HVS - despite its low sensitivity - only means that there is a vessel occlusion or highly reduced perfusion, which is seen far better on MRA and/or perfusion imaging and is also associated with more severe deficit, worse prognosis, positive DWI, PWI and MRA findings. This ends in the 'chicken-and-egg debate' about what is the relevant source of a problem, in this case strong predictors such as age, NIHSS, DWI lesion as well as vessel and perfusion status - or FLAIR HVS? What came first, the chicken or the egg? Eggs were around long before the first chicken arrived. They may, of course, not have been chicken eggs as we know them, but they were eggs.

\section{References}

1 Girot M, Gauvrit JY, Cordonnier C, Pruvo JP, Verdelho A, Leys D, Leclerc X: Prognostic value of hyperintense vessel signals on fluid-attenuated inversion recovery sequences in acute cerebral ischemia. Eur Neurol 2007;53:75-79.

\section{KARGER}

Fax +41613061234 E-Mail karger@karger.ch www.karger.com
(อ) 2007 S. Karger AG, Base

0014-3022/07/0572-0127\$23.50/0 
2 Schellinger PD, Chalela JA, Kang DW, Latour LL, Warach S: Diagnostic and prognostic value of early MR imaging vessel signs in hyperacute stroke patients imaged $<3$ hours and treated with recombinant tissue plasminogen activator. Am J Neuroradiol 2005;26:618-624.

3 Assouline E, Benziane K, Reizine D, Guichard JP, Pico F, Merland JJ, Bousser MG, Chabriat $\mathrm{H}$ : Intra-arterial thrombus visualized on $\mathrm{T}_{2}{ }^{*}$ gradient echo imaging in acute ischemic stroke. Cerebrovasc Dis 2005; 20:6-11.

4 Schellinger PD: Diagnostic accuracy and relevance of early MRI vessel signs are highly overrated. Cerebrovasc Dis 2006;21:215; author reply $215-216$.
5 Liebeskind DS: Location, location, location: angiography discerns early MR imaging vessel signs due to proximal arterial occlusion and distal collateral flow. Am J Neuroradiol 2005;26:2432-2433; author reply 2433-2434.

Peter D. Schellinger, MD, PhD

Department of Neurology, Schwabachanlage 6

DE-91054 Erlangen (Germany)

Tel. +499131853 4372, Fax +4991318534846

E-Mail peter.schellinger@neuro.imed.uni-erlangen.de 